\title{
AVALIAÇÃO DA EFICÁCIA E TOLERABILIDADE DA VIGABATRINA NA SÍNDROME DE WEST
}

\author{
Maria Helena P. Moraes ${ }^{1}$, Maria Augusta Montenegro², \\ Renata C. Franzon ${ }^{3}$, José O. Ávila4, Marilisa M. Guerreiro ${ }^{5}$
}

\begin{abstract}
RESUMO - A síndrome de West (SW) é uma epilepsia grave específica da infância, que se caracteriza pela tríade: espasmos em salvas, deterioração ou atraso neuropsicomotor e hipsarritmia ao eletrencefalograma. Objetivo:Avaliar a eficácia e segurança da vigabatrina (VGB) no tratamento da SW. Método: Foram sujeitos do estudo todos os pacientes com diagnóstico estabelecido de SW, que freqüentam ou freqüentaram o Ambulatório de Epilepsia Infantil do Hospital das Clínicas da UNICAMP, usam ou usaram VGB na tentativa de controlar as crises. Avaliamos sexo, idade, etiologia (sintomática ou criptogênica), doença(s) associada(s), idade do início dos espasmos, freqüência das crises antes e após o uso de VGB, achados de neuroimagem, EEG antes e depois do uso de VGB, medicações associadas, tempo para controle das crises, eletroretinograma, queixa visual após uso de VGB, efeitos adversos e história familial de epilepsia. Resultados: Foram avaliados 23 pacientes, sendo 16 do sexo masculino. A idade variou entre 1 ano e 3 meses a 11 anos e 5 meses (média $=5$ anos e 3meses). Dezesseis $(69,5 \%)$ pacientes apresentaram controle completo das crises, $5(22 \%)$ tiveram controle parcial e em $2(8,5 \%)$ pacientes os espasmos não foram controlados. Apenas uma paciente teve retinopatia gabaérgica. Seis pacientes $(26 \%)$ apresentaram eventos adversos - sonolência, agressividade ou retinopatia. Os pacientes com o início da SW após 6 meses de idade apresentaram melhor resposta à VGB $(p<0,05)$. Não houve diferença na resposta ao tratamento quanto ao tempo de introdução da VGB ou à etiologia ( $p>0,05)$. Após o tratamento com VGB, nenhum EEG apresentou hipsarritmia e $50 \%$ nomalizaram. Conclusão - Apesar do risco de retinopatia gabaérgica, os resultados acima mostram que o uso da VGB no controle dos espasmos infantis se justifica em pacientes com SW.
\end{abstract}

PALAVRAS-CHAVE: síndrome de West, vigabatrina, hipasaritmia, retinopatia gabaérgica.

\section{Efficacy and tolerability of vigabatrin in West syndrome}

ABSTRACT - West syndrome (WS) is a severe epileptic encephalopathy of childhood, characterized by spasms, developmental deterioration and hipsarhythymia. Objective: To evaluate the safety and efficacy of vigabatrin (VGB) in the treatment of WS. Method: We evaluated every patient diagnosed with WS seen at the pediatric epilepsy clinic and exposed to VGB. Patients were interviewed according to a semistructured questionnaire and we analyzed gender, age, etiology (cryptogenic or symptomatic), associated diseases, age of seizure onset, neuroimaging findings, EEG prior and after VGB, use of other antiepileptic drugs, time for seizure control, electro retinogram, visual complaints, adverse events and family history of epilepsy. Results. Twenty-three patients were evaluated, 16 boys, ages ranging from 1.25 years to 11.5 years $($ mean $=5 \mathrm{y} 3 \mathrm{~m})$. Sixteen $(69.5 \%)$ patients were seizure free, five $(22 \%)$ had partial seizure control and in two $(8.5 \%)$ there was no improvement. Only one patient presented gabaergic retinopathy. Six (26\%) patients p resented adverse events: somnolence, aggressivity or retinopathy. Patients with seizure onset after 6 months of age presented better results after VGB introduction $(p<0.05)$. There was no difference in seizure control according to duration of epilepsy before VGB treatment or etiology of the seizures $(p>0.05)$. After VGB, no patient presented hipsanhythymia and $50 \%$ had a normal EEG. Conclusion: Although VGB may be associated with serious adverse events such as gabaergic retinopathy, our results show that it should be considered in the treatment of WS.

KEY WORDS: West syndrome, vigabatrin, gabaergic retinopathy, hipsarhythymia.

Epilepsia é um grupo de doenças que se caracterizam por crises epilépticas recorrentes, na ausência de condições tóxico-metabólicas ou febris. A síndro- me de West (SW) é uma epilepsia grave específica da infância, que se caracteriza pela tríade: espasmos em salvas, deterioração ou atraso neuropsicomotor

Departamento de Neurologia, Faculdade de Ciências Médicas, (FCM), Universidade Estudual de Campinas (UNICAMP), Campinas SP, Brasil: ' ${ }^{A}$ luna de Medicina; ${ }^{2}$ Professora Doutora; ${ }^{3}$ Pós-Graduanda; ${ }^{4}$ Médico Assistente Doutor; ${ }^{5}$ Professora Associada.

Recebido 6 Setembro 2004, recebido na forma final 7 Dezembro 2004. Aceito 11 Fevereiro 2005.

Dra. Marilisa M. Guerreiro - Departamento de Neurologia - FCMIUNICAMP - Caixa Postal 6111 - $13083-970$ Campinas SP - E-mail: mmg@fcm.unicamp.br 
e hipsarritmia ao eletrencefalograma (EEG), manifestando-se principalmente antes do primeiro ano de vida. Existem variações em sua apresentação clínica, as quais parecem depender principalmente da etiologia. A freqüência das crises diminui com o passar da idade, sendo raras após os cinco anos. O curso da doença é variável. A SW está comumente associada a mau prognóstico, sendo a precocidade do diagnóstico e do tratamento fatores possíveis mas controversos quanto ao melhor prognóstico ${ }^{1}$. Nos EUA, hormônios adrenocorticotróficos, corticóides, ácido valpróico e benzodiazepínicos constituem a terapia mais freqüentemente prescrita para SW, sendo a primeira droga a mais preferível. No entanto, a relativa alta incidência de efeitos adversos como hipertensão, irritabilidade e maior susceptibilidade a infecções torna limitada a sua utilização².

$D$ rogas GABAérgicas, como a vigabatrina (VGB) e a tiagabina, mostraram-se eficazes no controle das crises parciais e espasmos infantis, mas o dano que pode causar ao campo visual com comprometimento irreversível da retina tem limitado seu uso $^{3}$. A incidência encontrada de defeitos visuais varia de zero $^{2}$ a mais de $50 \%^{3-5}$. Destes, $2 / 3$ não apresentaram retinopatia grave. Apesar deste grave efeito adverso que pode ocorrer em vários pacientes, a VGB tem sido considerada, por muitos, como a droga de escolha no tratamento da SW6-19.

Por se tratar de assunto controverso, resolvemos investigar a nossa experiência no assunto. Assim, o objetivo deste estudo foi avaliar a eficácia e segurança da VGB no tratamento da SW, particularmente em relação à presença ou não de alterações do campo visual.

\section{MÉTODO}

Foram sujeitos do estudo todos os pacientes com diagnóstico estabelecido de SW, que freqüentam ou freqüentaram o Ambulatório de Epilepsia Infantil do Hospital das Clínicas da UNICAMP, usam ou usaram VGB na tentativa de controlar as crises, e cujos pais ou cuidadores assinaram termo de consentimento informado. Foram excluídos aqueles com diagnóstico duvidoso de epilepsia.

Os pacientes foram convocados para uma consulta no Ambulatório de Epilepsia Infantil e durante a entrevista os dados foram coletados, o exame neurológico re alizado e o questionário preenchido. Nesta consulta foi agendado o exame de eletroretinograma, realizado pelo setor de Potenciais Evocados do nosso hospital. Posteriormente, os dados foram submetidos à análise estatística.

O questionário preenchido consistia dos seguintes itens: sexo, idade, cor, etiologia (sintomática ou criptogênica), doença(s) associada(s), idade do início dos espasmos, freqüência das crises antes e após o uso de VGB, achados na tomografia computadorizada (CT) e na ressonância magnética (RM), achados ao EEG (presença de hipsarritmia) antes e depois do uso de VGB, VGB como primeira opção de tratamento, medicações associadas, tempo de crise até início de VGB, dose, tempo para controle das crises, eletroretinograma, queixa visual após uso de VGB, alteração do comportamento e do sono após o uso de VGB, outro efeito adverso e história familial de epilepsia.

O protocolo do nosso serviço orienta a introdução da VGB na dose de $100 \mathrm{mg} / \mathrm{kg} / \mathrm{d}$. Se em duas semanas não houver resposta, a droga deverá ser suspensa. Havendo controle completo das crises, a medicação é mantida por seis meses. Se a resposta terapêutica estiver entre 50 e $100 \%$, a dose da VGB poderá ser aumentada para 150 $\mathrm{mg} / \mathrm{kg} / \mathrm{d}$ ou a associação com outra droga poderá ser considerada.

O trabalho foi submetido e aprovado pelo Comitê de Ética da nossa instituição.

\section{RESULTADOS}

Foram avaliados 23 pacientes, sendo 16 do sexo masculino. A idade variou entre 1 ano e 3 meses a 11 anos e 5 meses (média = 5anos e 3meses). Dezesseis $(69,5 \%)$ pacientes apresentaram controle completo das crises, $5(22 \%)$ tiveram controle parcial e em $2(8,5 \%)$ pacientes os espasmos não foram controlados (Fig 1). As características clínicas dos pacientes e os exames complementares constam das Tabelas 1 e 2 .

Apenas uma paciente apresentou ao exame de eletroretinograma ausência de potenciais oscilatórios bilaterais compatíveis com retinopatia gabaérgica. Outros seis pacientes apresentaram outras alterações retinianas: retinopatia da prematuridade (2); alteração retiniana prévia à introdução da VGB (1); comprometimento retiniano unilateral (1); e a usência de resposta dos fotorreceptores (2).

Seis pacientes $(26 \%)$ apresentaram eventos adversos - sonolência, agressividade ou retinopatia. Os pacientes com o início da SW após 6 meses de idade apresentaram melhor resposta à VGB $(p<0,05)$. Não houve diferença na resposta ao tratamento quanto ao tempo de introdução da VGB ou à etiologia $(p>0,05)$.

O EEG era anormal e apresentava hipsarritmia em quase todos os pacientes antes do tratamento, exceto no paciente número 3 que apresentava atividade epileptiforme multifocal. Quatorze crianças realizaram o EEG tanto antes quanto após o tratamento e a figura 2 mostra a evolução dos achados. Após o tratamento com VGB, nenhum EEG apresentou hipsarritmia e 50\% normalizaram. Treze de 14 mães interrogadas informaram haver aquisições neuropsicomotoras no decorrer do tratamento com VGB. 
Tabela 1. Características clínicas dos pacientes do estudo.

\begin{tabular}{|c|c|c|c|c|c|}
\hline Paciente & Inic SW & Inic VGB & Etiologia & Freq antes & Freq após \\
\hline 1 & 1a $8 m$ & $5 a 6 m$ & $\mathrm{EHI}$ & diárias & diárias \\
\hline 2 & $6 m$ & $6 m$ & ET & diárias & 0 \\
\hline 3 & $1 \mathrm{~m}$ & $2 \mathrm{~m} \mathrm{17d}$ & $\mathrm{ET}$ & 2 & 0 \\
\hline 4 & $3 m$ & $4 m$ & $\mathrm{EHI}$ & 3 & 1 \\
\hline 5 & $5 m$ & $7 m$ & $\mathrm{EHI}$ & diárias & 0 \\
\hline 6 & $3 m$ & $9 m$ & Criptogênica & 8 & 1 \\
\hline 7 & $4 m 20 d$ & $5 m$ & Criptogênica & 6 & 0 \\
\hline 8 & $7 m$ & $9 m$ & $\mathrm{EHI}$ & 1 & 0 \\
\hline 9 & $3 m$ & $9 m$ & MF do SNC & 50 & 0 \\
\hline 10 & $8 m$ & $2 a$ & Criptogênica & 60 & 0 \\
\hline 11 & $4 m$ & $11 \mathrm{~m} \mathrm{20d}$ & Facomatose & diárias & 0 \\
\hline 12 & $3 m$ & $2 a 3 m$ & $\mathrm{EHI}$ & 10 & 0 \\
\hline 13 & $1 \mathrm{~m}$ & $3 a$ & MF do SNC, EHI & diárias & diárias \\
\hline 14 & $5 m$ & $6 m 23 d$ & Neurofibromatose I & diárias & 0 \\
\hline 15 & $6 m$ & 1a $1 \mathrm{~m}$ & $\mathrm{EHI}$ & 7 & 0 \\
\hline 16 & $9 m$ & 1a $1 \mathrm{~m}$ & ET & 8 & 0 \\
\hline 17 & $7 m$ & $8 m$ & Criptogênica & 10 & 0 \\
\hline 18 & $9 m$ & $9 m$ & $\mathrm{EHI}$ & 3 & 0 \\
\hline 19 & $7 m$ & $7 m$ & $\mathrm{ET}$ & 20 & 0 \\
\hline 20 & $4 m$ & $1 \mathrm{a} 3 \mathrm{~m}$ & Criptogênica & 12 & 0 \\
\hline 21 & $3 m$ & $11 \mathrm{~m}$ & Lisencefalia & diárias & $1 /$ mês \\
\hline 22 & $5 m$ & $1 \mathrm{a}$ & $\mathrm{EHI}$ & 15 & 5/dia \\
\hline 23 & $8 m$ & $5 a 6 m$ & ET & diárias & 1/semana \\
\hline
\end{tabular}
tratamento; EHI, encefalopatia hipóxico-isquêmica; ET, esclerose tuberosa; MF do SNC, malformação do sistema nervoso central.

Tabela 2. Exames complementares.

\begin{tabular}{|c|c|c|c|c|c|c|c|}
\hline $\begin{array}{l}\text { Hipsar. } \\
\text { antes }\end{array}$ & $\begin{array}{l}\text { EEG } \\
\text { após }\end{array}$ & $\begin{array}{l}\text { Hipsar. } \\
\text { após }\end{array}$ & $\begin{array}{c}\text { T. crise até } \\
\text { VGB }\end{array}$ & T. controle & $\mathrm{CT}$ & RNM & ERG \\
\hline $\operatorname{sim}$ & sem & - & & Não houve & alt. & alt. & sem \\
\hline $\operatorname{sim}$ & $\mathrm{nl}$ & não & $<1 \mathrm{~m}$ & $<7 d$ & $\mathrm{nl}$ & sem & alt. \\
\hline não & sem & não & $1 \mathrm{~m} \mathrm{17d}$ & $<7 d$ & alt & sem & $\mathrm{nl}$ \\
\hline $\operatorname{sim}$ & $\mathrm{nl}$ & não & $<1 \mathrm{~m}$ & & alt. & sem & sem \\
\hline $\operatorname{sim}$ & alt. & não & $2 m$ & $<7 d$ & alt. & sem & sem \\
\hline $\operatorname{sim}$ & sem & - & $6 m$ & $10 d$ & sem & $\mathrm{nl}$ & $\mathrm{nl}$. \\
\hline $\operatorname{sim}$ & $\mathrm{nl}$ & não & $10 \mathrm{~d}$ & & $\mathrm{nl}$ & sem & $\mathrm{nl}$ \\
\hline $\operatorname{sim}$ & alt. & não & $2 m$ & & alt. & sem & alt. \\
\hline $\operatorname{sim}$ & sem & - & $4-5 m$ & $10 d$ & alt. & alt. & sem \\
\hline $\operatorname{sim}$ & $\mathrm{nl}$ & não & $1 \mathrm{a} 4 \mathrm{~m}$ & $<1 \mathrm{~m}$ & $\mathrm{nl}$ & sem & $\mathrm{nl}$ \\
\hline $\operatorname{sim}$ & sem & - & $7 m$ & $2 m$ & alt. & sem & sem \\
\hline $\operatorname{sim}$ & sem & - & $2 a$ & $1 \mathrm{~m}$ & alt. & sem & alt. \\
\hline $\operatorname{sim}$ & alt. & não & $3 a$ & Não houve & alt. & sem & $\mathrm{nl}$ \\
\hline $\operatorname{sim}$ & $\mathrm{nl}$ & não & $1 \mathrm{~m} \mathrm{15d}$ & $15 d$ & $\mathrm{nl}$ & sem & sem \\
\hline $\operatorname{sim}$ & sem & - & $7 m$ & $<7 d$ & alt. & sem & alt. \\
\hline $\operatorname{sim}$ & alt. & não & $5 m$ & $2 m$ & alt. & sem & sem \\
\hline $\operatorname{sim}$ & alt. & não & $4 m$ & $<14 d$ & sem & $\mathrm{nl}$ & sem \\
\hline $\operatorname{sim}$ & $\mathrm{nl}$ & não & $4 d$ & $3 d$ & sem & sem & sem \\
\hline $\operatorname{sim}$ & $\mathrm{nl}$ & não & $5 d$ & $>1 \mathrm{~m}$ & sem & alt. & alt. \\
\hline $\operatorname{sim}$ & alt. & não & $11 \mathrm{~m}$ & $14 d$ & alt. & sem & alt. \\
\hline $\operatorname{sim}$ & sem & - & $8 m$ & $7 d$ & alt. & sem & $\mathrm{nl}$ \\
\hline $\operatorname{sim}$ & sem & - & $7 m$ & & alt. & sem & alt. \\
\hline $\operatorname{sim}$ & alt. & não & $4 \mathrm{a} 10 \mathrm{~m}$ & & alt. & sem & $\mathrm{nl}$ \\
\hline
\end{tabular}




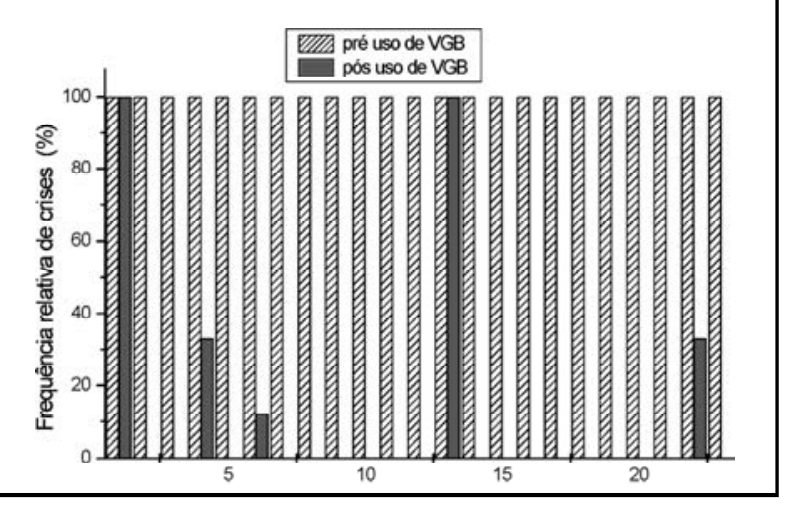

Fig 1. Freqüência relativa de crises antes e após o uso de viga batrina.

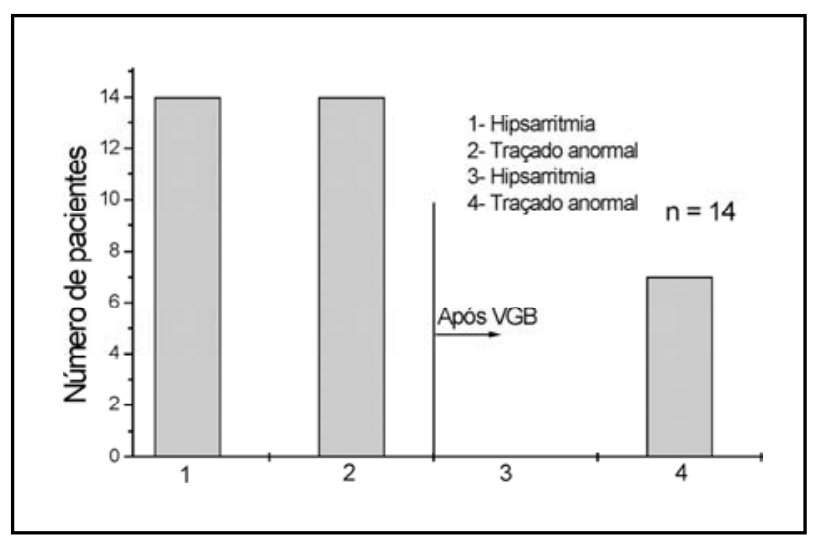

Fig 2. Evolução do eletrencefalograma.

\section{DISCUSSÃO}

A VGB é eficaz no controle dos espasmos infantis. Em nosso estudo, quase $70 \%$ dos pacientes obtiveram controle completo das crises epilépticas. Sabe-se que o prognóstico da SW está relacionado ao controle dos espasmos e quanto mais precoce e efetivo o tratamento, tanto melhor será o desenvolvimento neuropsicomotor da criança. Desde as primeiras descrições do sucesso terapêutico da SW com a VGB, particularmente quando a etiologia é esclerose tuberosa ${ }^{6,7}$, a eficácia dessa droga tem sido comprovada e enfatizada por outros estudos ${ }^{8-19}$. Vários desses autores concluíram que a VGB deve ser considerada como a droga de primeira escolha no tratamento da SW não só pela eficácia igual ou maior que ACTH, como também pelo menor índice de efeitos colaterais graves. Em nosso estudo, os pacientes com o início da SW após 6 meses de idade a p resentaram melhor resposta à VGB. Sabe-se que o prognóstico é mais favorável quando o início dos espasmos é mais tardio e isto provavelmente está co rrelacionado com a etiologia que é um dos principais fatores determinantes do prognóstico'. Assim, a melhor resposta terapêutica obtida em nossos pacientes deve, em última análise, estar relacionada à etiologia.

Quanto à tolerabilidade à VGB, excetuando-se a retinopatia gabaérgica que será abordada a seguir, os principais eventos adversos são sonolência, irritabilidade, agitação e ganho de peso. Esses efeitos

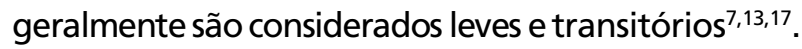

A questão da retinopatia gabaérgica causada pela VGB tem sido motivo de inúmeros relatos. A disfunção periférica dos cones da retina levando a constrição do campo visual está bem estabeleci$\mathrm{da}^{20,21}$, enquanto não está claro se também pode haver comprometimento da função visual central ${ }^{22}$. O percentual da alteração de campo visual varia de acordo com cada estudo e com os métodos de investigação empregados chegando a $69 \%$ de positividade de defeito bilateral de campo visual ${ }^{23}$. Ent retanto, o tempo de exposição à VGB e a dose total utilizada parecem ser fatores determinantes ${ }^{24,27}$, e, em um estudo com altos índices de dados alterados ${ }^{5}$, os pacientes estavam em uso da VGB há pelo menos três anos. Isto tem encorajado alguns autores a reduzirem o tempo de tratamento e há a proposta de não se prolongar o tratamento da SW por mais de seis meses $^{28}$, o que possivelmente reduziria a chance do comprometimento visual. Além do tempo de uso da droga e da dose utilizada, parece ainda haver diferença entre adultos e crianças em relação à chance de problemas visuais, pois os trabaIhos realizados na infância apontam para taxas me$n$ o res de danos causados pela VGB ${ }^{27,29}$. Outra questão em aberto é a reversibilidade ou não da retinopatia gabaérgica. Enquanto alguns afirmam que as anormalidades persistem ${ }^{23,30}$, outros apontam para o fato alentador da possível reversão da constrição do campo visual ${ }^{31-33}$.

Apenas um de nossos pacientes apresentou alteração ao eletroretinograma compatível com retinopatia gabaérgica. Esta criança estava em uso da VGB há cinco meses e já havíamos iniciado a retirada gradual da droga quando o exame foi realizado. Atualmente ela se encontra totalmente controlada dos espasmos e sem medicação há dois meses. Pretendemos repetir o eletroretinograma em alguns meses e esperamos que os dados alterados não se mantenham.

Apesar do risco de retinopatia gabaérgica, os resultados acima mostram que o uso da VGB no controle dos espasmos infantis se justifica em pacientes com SW. O nosso trabalho deixa claro que o risco de comprometimento do campo visual é pe- 
queno em crianças em uso criterioso da VGB e que o benefício do tratamento é grande. Em outras palavras, os nossos dados reforçam a maioria das opiniões vigentes sobre o assunto. Além disso, o medo desmesurado da retinopatia gabaérgica tem feito com que a VGB não seja muito indicada. Este receio nem sempre se justifica, pois se houver cautela no uso da VGB e acompanhamento próximo do paciente com a realização de eletroretinogramas, a medicação pode e deve continuar a ser utilizada nesta grave condição clínica que é a síndrome de West.

\section{REFERÊNCIAS}

1. Appleton RE. West syndrome: long-term prognosis and social aspects. Brain Dev 2001;23:688-691.

2. Mitchell WG, Shah NS. Vigabatrin for infantile spasms. Pediatr Neurol 2002;27:161-164.

3. Wallace SJ. Newer antiepileptic drugs: advantages and disadvantages; Brain \& Dev 2001;23:277-283.

4. Harding GF, Wild JM, Robertson KA, Rietbrock S, Martinez C. Separating the retinal electrophysiologic effects of vigabatrin: treatment versus field loss. Neurology 2000;55:347-352.

5. McDonagh J, Stephen LJ, Dolan FM, et al. Peripheral retinal dysfunction in patients taking vigabatrin. Neurology 2003;61:1690-1694.

6. Chi ronC, Dulac O, Luna D, et al. Vigabatrin in infantile spasms. Lancet 1990;335:363-364.

7. Chiron C, Dulac O, Beaumont D, Palacios L, Pajot N, Mumford J. Therapeutic trial of vigabatrin in refractory infantile spasms. J Child Neurol 1991;(Suppl 2):S52-S59.

8. Rotta NT, Silva AR, Ohlweiler L, Riesgo R. Vigabatrin in the treatment of epilepsy in patients with West syndrome and tuberous sclerosis. Arq Neuropsiquiatr 2003;61:988-990.

9. Curatolo P, Seri S, Verdecchia M, Bombardieri R. Infantile spasms in tuberous sclerosis complex. Brain Dev 2001;23:502-507.

10. Elterman RD, Shields WD, Mansdield KA, Nakagawa J US Infantile Spasms Vigabatrin Study Group. Randomized trial of vigabatrin in patients with infantile spasms. Neurology 2001;57:1416-1421

11. Riikonen RS. Steroids or vigabatrin in the treatment of infantile spasms? Pediatr Neurol, 2000;23:403-408.

12. Antoniuk SA, Bruck I, Spessatto A, et al. West syndrome: clinical and electroencephalographic follow up of 70 patients and response to its treatment with adrenocorticotropic hormone, prednisone, vigabatrin, nitrazepam and valproate. Arq Neuropsiquiatr 2000;58:683-690.

13. Fejerman N, Cersosimo R, Caraballo R, et al. Vigabatrin as a firstchoice drug in the treatment of West syndrome. J Child Neurol. 2000; 15:161-165.

14. Appleton RE, Peters AC, Mumford JP, Shaw DE. Randomised, placebo- controlled study of vigabatrin as first-line treatment of infantile spasms. Epilepsia 1999;40:1627-1633.

15. Granstrom Ml, Gaily E, Liukkonen E. Treatment of infantile spasms of a population-based study with vigabatrin as the first drug for spasms. Epilepsia 1999;40:950-957.

16. Cossette P, Riviello JJ, Carmant L. ACTH versus vigabatrin therapy in infantile spasms: a retrospective study. Neurology 1999;52:1691-1694.

17. Villeneuve N, Soufflet C, Plouin P, Chiron C, Dulac O. Treatment of infantile spasms with vigabatrin as first-line therapy and in monoteraphy: apropos of 70 infants. Arch Pediatr 1998;5:731-738.

18. Wohlrab G, Boltshauser E, Schmitt B. Vigabatrin as a first-line drug in West syndrome: clinical and electroencephalographic outcome. Neuropediatrics 1998;29:133-136.

19. Vigevano F, Cilio MR. Vigabatrin versus ACTH as first-line treatment for infantile spasms: a randomized, prospective study. Epilepsia 1997; 38:1270-1274.

20. Arndt CF, Derambure P, Defoort-Dhellemmes S, Hache JC. Outer retinal dysfunction in patients with vigabatrin. Neurology 1990;52:1201-1205.

21. Krauss GL, Johnson MA, Miller NR. Vigabatrin-associated retinal cone system dysfunction: electroretinogram and ophthalmologic findings. Neurology 1998;50:614-618.

22. Banin E, Shalev RS, Obolensky A, Neis R, Chowers I, Gross-Tsur V. Retinal function abnormalities in patients treated with vigabatrin. Arch Ophthalmol 2003;121:811-816.

23. Harding GF, Wild JM, Robertson KA, et al. Electro-oculography, electroretinography, visual evoked potentials, and multifocal electro retinography in patients with vigabatrin-attributed visual field constriction. Epilepsia 2000;41:1420-1431.

24. Malmgren K, Ben-Menachem E, Frisen L. Vigabatrin visual toxicity: evolution and dose dependence. Epilepsia 2001;42:609-615.

25. Hardus P, Ve rduin WM, Engelsman M, et al. Visual field loss associated with vigabatrin: quantification and relation to dosage. Epilepsia 2001; 42:262-267.

26. Jensen H, Sjo O, Uldall P, et al. Vigabatrin and retinal changes. Doc Ophthalmol 2002;104:171-180.

27. Schmitz B, Schmidt T, Jokiel B, Pfeiffer S, Tiel-Wilck K, Ruther K. Visual field constriction in epilepsy patients treated with vigabatrin and other antiepileptic drugs: a prospective study. J Neurol 2002;249:469-475.

28. CapovillaG, Beccaria F, Montagnini A, et al. Short-term nonhormonal and nonsteroid treatment in West syndrome. Epilepsia 2003;44:1085-1088.

29. Koul R, Chacko A, Ganesh A, Bulusu S, Al Riyami K. Vigabatrin associated retinal dysfunction in children with epilepsy. Arch Dis Child 2001; 85:469-473.

30. Schmidt T, Ruther K, Jokiel B, Pfeiffer S, Tiel-Wilck, Schmitz B. Is visual field constriction in patients treated with vigabatrin reversible? J Neurol 2002;249:1066-1071.

31. Giordano L, Valseriati D, Vignoli A, Morescalchi F, Gandolfo E. Another case of reversibility of visual-field defect induced by vigabatrin monotherapy: is young age a favorable factor? Neurol Sci 2000;21:185-186.

32. Versino M, Veggiotti P. Reversibility of vigabatrin-induced visual-field defect. The Lancet 1999;354:486.

33. Vanhatalo S, Alen R, Riikonen R, et al. Reversed visual field constrictions in children after vigabatrin withdrawal - true retinal recovery or improved test performance only? Seizure 2001;10:508-511. 